\title{
The role of multi-parametric magnetic resonant imaging as a non invasive biomarker for differentiation between different prostatic lesions
}

\author{
Youssef AT (1), Aboelnor MI (2) and Zahran AA (3)
}

1. Assistant professor of Radiodiagnosis, Faculty of Medicine - Fayoum University.

2. Lecturer of Radiodiagnosis, Faculty of Medicine - Fayoum University.

3. Department of Radiodiagnosis, Faculty of Medicine - Fayoum University

Corresponding Author Name: Ahmed Ayman Mahmoud Mohamed Zahran

Department of Radiodiagnosis Faculty of Medicine, Fayoum University

E-Mail: dr_aaz@yahoo.com

Telephone: $+\mathbf{2 0 1 0 6 4 8 3 9 9 1 7}$

\begin{abstract}
Purpose to assess the value of the multi-parametric MRI of prostate as a non invasive biomarker in differentiation between different prostatic lesions. Patients and Methods: Prospective study was done on 30 male patients complaining from lower urinary tract symptoms with elevated prostatitic specific antigen ( PSA ) level or abnormal digital rectal examination ( DRE ). Results: MRI was good positive test in diagnosis of malignancy with sensitivity $(100 \%)$ and specificity $(81.1 \%)$ also it consider good positive for diagnosis of isolated benign prostatic hyperplasia (BPH) with sensitivity (89\%) and specificity (80\%). Conclusions: Prostate imaging reporting and data system. (PIRADS) is an efficient system for the diagnosis of different prostatic disease and depending on all sequences give better results than relying in the diagnosis on individual sequences.
\end{abstract}

\section{KEYWORDS}

Cancer Prostate, BPH, Prostatitis, PIRADS, PSA, Gleason Score.

\section{INTRODUCTION}

Prostatic lesions are very common findings which include Benign prostatic hyperplasia, Prostatitis and prostatic cancer (Which is the 2nd most common cancer worldwide for males, and the 5 th most common cancer overall $14 \%$ of the total in males and $7 \%$ of the total overall ) (1)

MR imaging is nowadays playing a role in the clinical pathways of detection, local staging, active surveillance, and post treatment follow-up. By increasing influence that MR imaging has on clinical decision making, it is important to be aware of the potential pitfalls in imaging interpretation (2)

Multi-parametric MRI (mpMRI) combines anatomic $\mathrm{T} 2 \mathrm{~W}$ with functional and physiological assessment, including diffusion weighted imaging 
(DWI) and its derivative apparent diffusion coefficient (ADC) maps and dynamic contrast enhanced (DCE) MRI. mpMRI, have substantially improved diagnostic capabilities for addressing the central challenges in prostate diseases and lead to improving accuracy of diagnosis, Prostate
Imaging Reporting and Data System (PI-RADS) is a scoring system that aims to enable consistent interpretation, communication and reporting of prostate multi-parametric MRI (mpMRI) findings.

\section{PATINETS AND METHODS}

This study was reviewed and approved by the Faculty of Medicine Research Ethical Committee. The researcher informed the participants about the objectives of the study, the examination, investigation that was done. Also the confidentiality of their information and their right not to participate in the study.

Prospective cross-sectional study was performed and data were collected and coded to facilitate data manipulation and double entered into Microsoft Access and data analysis was performed using Statistical Package of Social Science (SPSS) software version 18 in windows 7. Simple descriptive analysis in the form of numbers and percentages for qualitative data, and arithmetic means as central tendency measurement, standard deviations as measure of dispersion for quantitative parametric data. Sensitivity and specificity test for testing a new test with ROC curve "Receiver Operating Characteristic". The P-value $\leq 0.05$ was considered the cut-off value for significance.

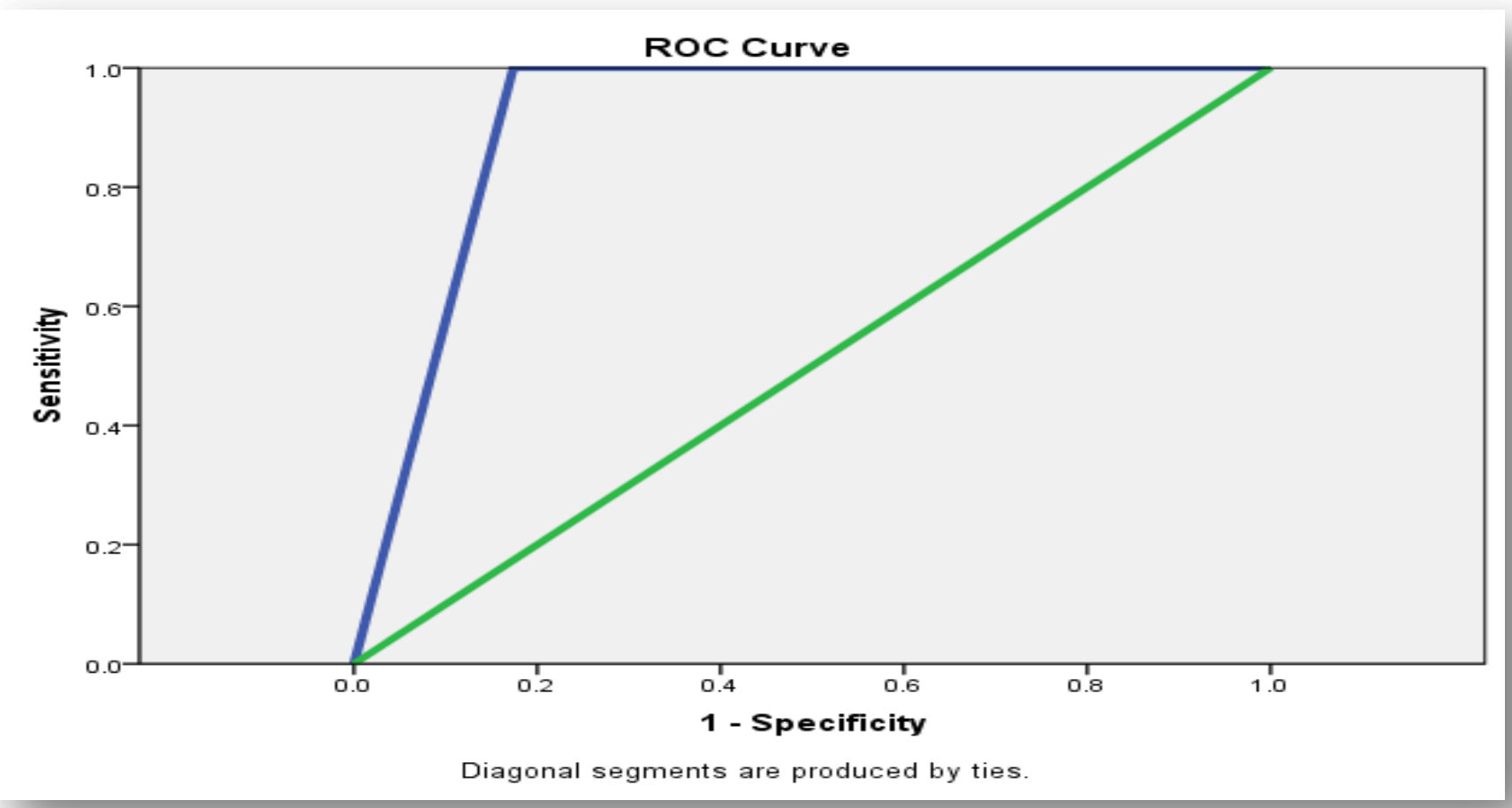

Our study was performed from September 2018 to February 2019. 
The patients with contraindications to MR imaging as ( cardiac pacemaker, Aneurysmal clips, bone growth stimulators, electrically programmed infusion pump ,bullets and shrapnel ,orthopedic implants and devices , intravascular stents, coils and filters) were excluded. The patients who refuse to participate in the study were also excluded.

MR imaging was performed with a 1.5 Tesla magnet .Titan closed machine (Toshiba medical systems). Cases were imaged in the supine position using pelvic phased- array surface coil with different scanning protocols as follows:-

\section{Detection protocol}

T2WI axial+sagittal: $4 \mathrm{~mm}$ at $1.5 \mathrm{~T}$; in plane resolution: $0.5 \times 0.5 \mathrm{~mm}$ to $0.7 \times 0.7 \mathrm{~mm}$ at $1.5 \mathrm{~T}$. DWI axial: $5 \mathrm{~mm}$ at $1.5 \mathrm{~T}$, in-plane resolution: $1.5 \times 1.5 \mathrm{~mm}$ to $2.0 \times 2.0 \mathrm{~mm}$ at $1.5 \mathrm{~T}$.

DCE-MRI axial: $4 \mathrm{~mm}$ at $1.5 \mathrm{~T}$ in plane resolution: $1.0 \times 1.0 \mathrm{~mm}$ at $1.5 \mathrm{~T}$.

Staging protocol ( In suspected Cancer prostate )

45-min protocol for evaluating minimal extra-capsular extension. Images should include entire prostate, with anti-peristaltic drugs. T2WI axial, coronal and sagittal planes, $3 \mathrm{~mm}$ at $1.5 \mathrm{~T}$ and in plane resolution: $0.3 \times 0.3 \mathrm{~mm}$ to $0.7 \times 0.7 \mathrm{~mm}$ at $1.5 \mathrm{~T}$. DWI and DCE as detection protocol.

\section{Nodes and bone protocol ( In Cancer prostate )}

30-min protocol, to assess nodal size and bone marrow metastases. Should be performed separately from $\mathrm{A}$ and $\mathrm{B}$, as most patients do not require bone or node staging. T1WI coronal of lower lumbar spine plus pelvis (SE or f/T SE) 3.0-mm slices. 3D f/T SE T2WI coronal of lower lumbar spine plus pelvis; $1.0-\mathrm{mm}$ isometric voxels. DWI coronal of lower lumbar spine plus pelvis (b-values 0 and 600); slice thickness 3$4 \mathrm{~mm}$, in plane resolution: $2.5-3.0 \mathrm{~mm}$ voxels. T1WI sagittal cervical and thoracic spine (SE or f/T SE). STIR or DWI sagittal cervical and thoracic spine. (3)

PIRADS scoring diagram was used as follows:-

\begin{tabular}{|c|c|c|}
\hline $\begin{array}{l}\text { PI-RADS } \\
\text { classification }\end{array}$ & Definition & Total score with T2, DWI,DCE \\
\hline 1 & Most probably benign & 3,4 \\
\hline 2 & Probably benign & 5,6 \\
\hline 3 & Intermediate & $7-9$ \\
\hline 4 & Probably malignant & $10-12$ \\
\hline 5 & Highly suspicious for malignant & $13-15$ \\
\hline
\end{tabular}




\section{Peripheral Zone Lesion}

\section{DWI SCORE}
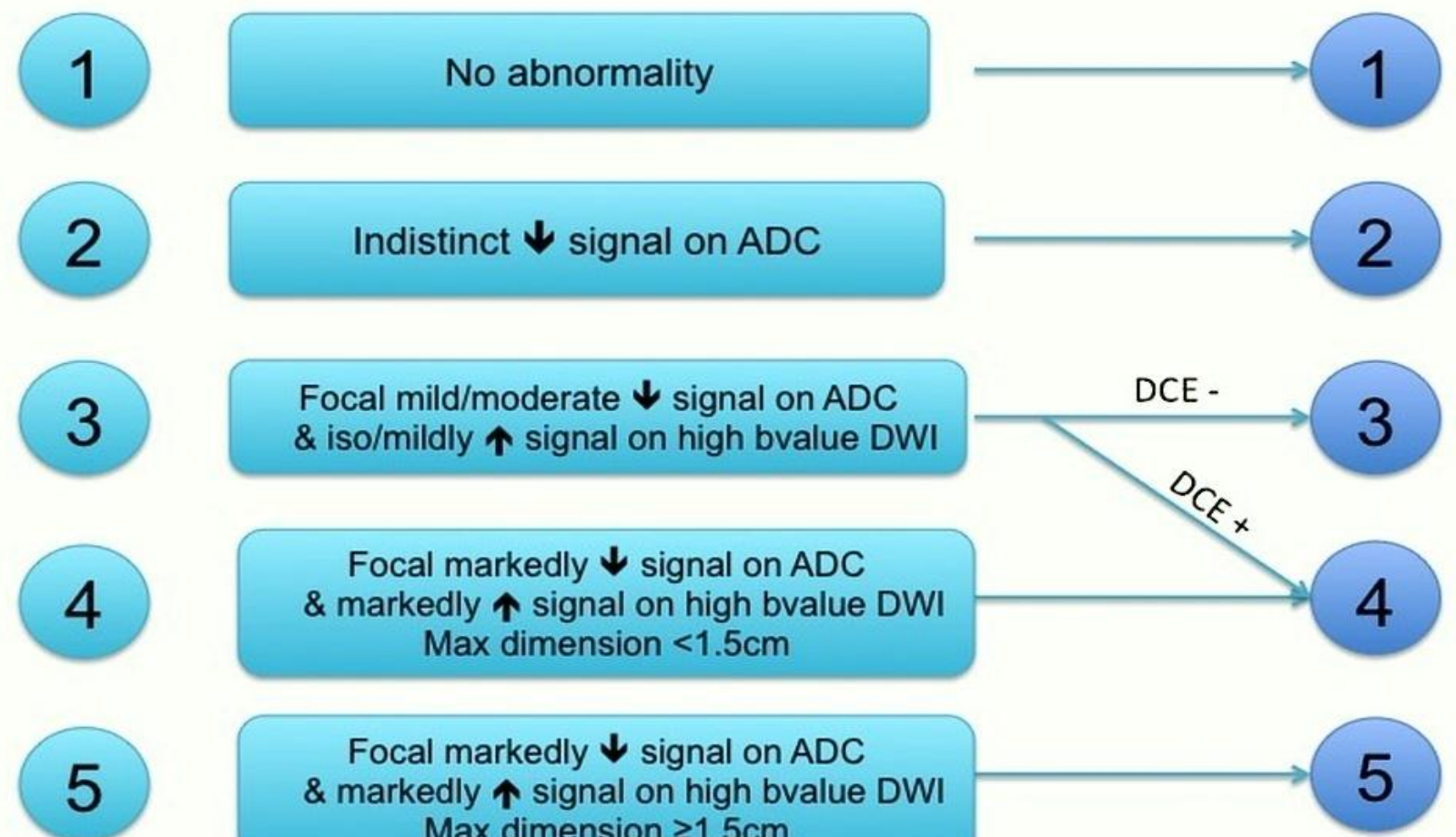

Focal mild/moderate $\downarrow$ signal on ADC

\& iso/mildly $\uparrow$ signal on high bvalue DWI

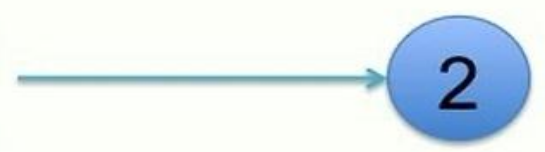

Focal markedly $\downarrow$ signal on ADC

\& markedly $\uparrow$ signal on high bvalue DWI Max dimension $<1.5 \mathrm{~cm}$

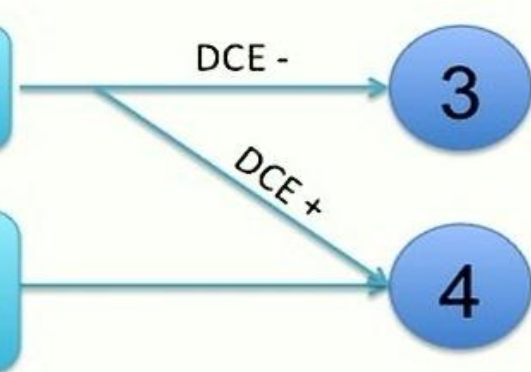

Focal markedly $\downarrow$ signal on ADC

$\&$ markedly $\uparrow$ signal on high bvalue DWI

Max dimension $\geq 1.5 \mathrm{~cm}$ 


\section{PIRADS}

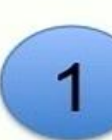

\section{2}

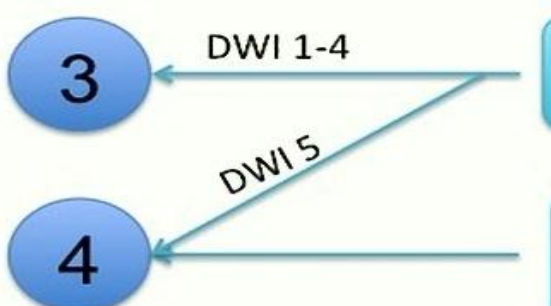

5

\section{Transitional Zone Lesion}

T2WI SCORE

No abnormality

Circumscribed hypointense or

heterogeneous nodules (BPH)

Heterogeneous signal intensity with obscured margins

Lenticular or non-circumscribed, homogeneous, moderately hypointense Max dimension $<1.5 \mathrm{~cm}$

\section{Lenticular or non-circumscribed,} homogeneous, moderately hypointense

Max dimension $\geq 1.5 \mathrm{~cm}$ OR invasive

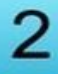

\section{3}

1

5

\section{RESULTS}

Our study included 30 male patients with different age groups ( from 55 to 85 years ) with elevated PSA, abnormal DRE and different lower urinary tract symptoms as (Urine retention, dysuria and hematuria ). Out of them 11 patients had findings suggestive of prostatic carcinoma on imaging findings, 19 patients suggestive of benign findings (BPH and prostatitis). 
$73.3 \%$ of patients had high PSA, $6.7 \%$ show borderline, versus $20 \%$ show normal PSA.

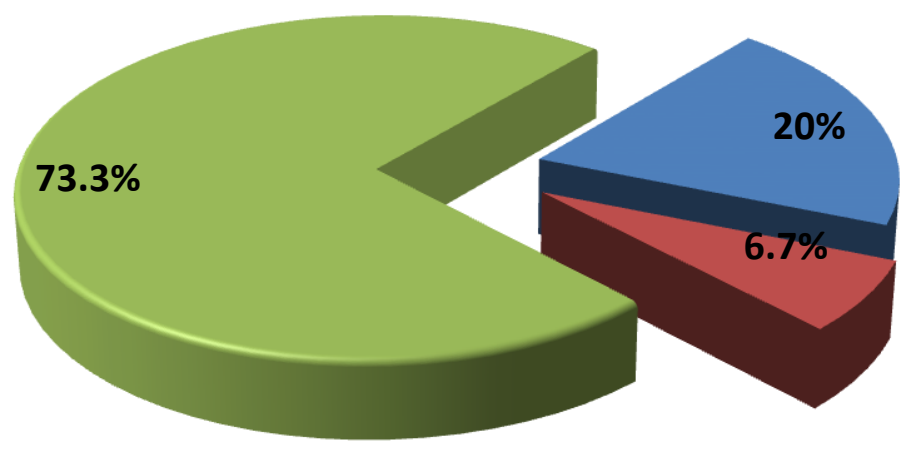

Normal Borderline $\mathbf{A}$ High

PSA among study group

In our study we made a side comparison between overall multi-parametric MRI findings and pathology findings that resulted in:

\begin{tabular}{|l|c|c|c|}
\hline \multirow{2}{*}{ Biopsy Proven } & \multicolumn{3}{|c|}{ Over all multi-parametric MRI } \\
\cline { 2 - 4 } & $\begin{array}{l}\text { BPH } \\
(\mathrm{N}=10)\end{array}$ & $\begin{array}{l}\text { Malignant } \\
(\mathrm{N}=11)\end{array}$ & $\begin{array}{l}\text { BPH \& } \\
\text { Prostatitis (N=9) }\end{array}$ \\
\hline BPH (N=16) & $8(80 \%)$ & $2(18.2 \%)$ & $6(66.7 \%)$ \\
\hline Malignant (N=9) & $0(0 \%)$ & $9(81.1 \%)$ & $0(0 \%)$ \\
\hline BPH \& Prostatitis (N=5) & $2(20 \%)$ & $0(0 \%)$ & $3 \quad(33.3 \%)$ \\
\hline
\end{tabular}

The correlation between PIRADS and Gleason grading couldn't be fully expressed and show variability in its results although high values of grade V showed high risk in Gleason grades but also remains 38.4\% showed grade V in PIRADS and moderate Gleason grades. 75\% of grade IV PIRADS scoring (clinical significant cancer is likely to be present) showed Gleason $6 \& 7$ stages which represents moderate risk. Only $25 \%$ of grade V PIRADS scoring (clinical significant cancer is highly likely to be present) showed Gleason 8 stage which represents moderate risk. $61.54 \%$ of grade $\mathrm{V}$ (clinical significant cancer is highly likely to be present) showed Gleason $8 \& 9$ stages which represents high grades. While $38.46 \%$ of grade V (clinical significant cancer is highly likely to be present) showed Gleason $6 \& 7$ stages which represents high grades.

Concerning central and transitional zone benign prostatic hyperplasia changes and possibility of presence central and transitional zone carcinoma the results showed specificity $47.7 \%$ for grade II and $42.1 \%$ for 
grade III. The individual T2W PIRADS analysis for carcinoma cases showed specificity $28.5 \%$ for diagnosis of equivocal carcinoma grade III and $28.5 \%$ for grade IV and $43 \%$ for grade V. Higher specificity results on combining T2W and diffusion PIRADS analysis showing that $28.5 \%$ for diagnosis of grade IV and $71.5 \%$ for grade V. Overall PIRADS analysis for carcinoma cases showed specificity $28.5 \%$ for diagnosis of grade IV and $71.5 \%$ for grade V.

T2WI of $63.3 \%$ of cases show iso- intensity but $23.3 \%$ had hypo-intensity, and $6.7 \%$ with hyper-intensity, but $6.7 \%$ show mixed intensity. Diffusion weighted imaging ( DWI ) \&Apparent diffusion coefficient (ADC ) $23.3 \%$ were restricted but $76.7 \%$ were not restricted. Dynamic contrast enhancement ( DCE ) finding $80 \%$ had no early enhancement, versus $20 \%$ had early enhancement. $44.4 \%$ of cancer patients have moderate to high Gleason score grade and $55.6 \%$ of cancer patients have low Gleason score grade.
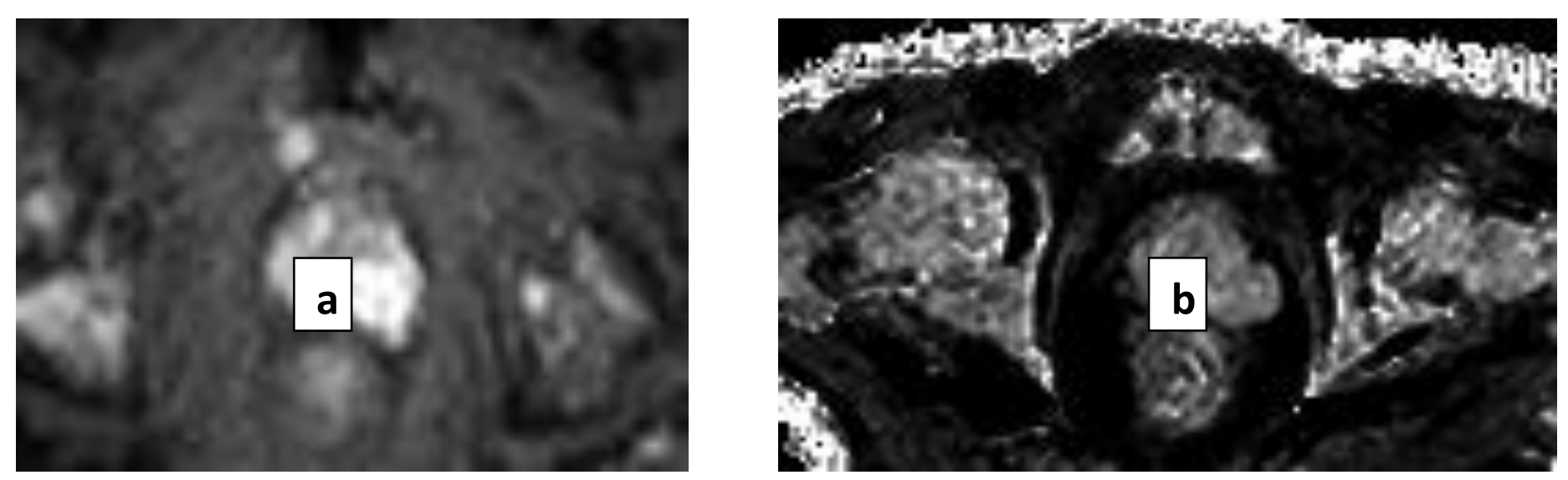

This figure of male patient Seventy Four years old presented with increased PSA level and gross painless hematuria. MRI prostate ( Diffusion and ADC mapping sequences ) shows restricted pattern of diffusion PIRADS grade $V$ of Left peripheral zone focal lesion. This lesion is pathologically proven to be prostatic adenocarcinoma Gleason grade 8.

MRI was good positive test in diagnosis of malignancy with sensitivity (100\%) and specificity (81.1\%) also it consider good positive for diagnosis of isolated BPH with sensitivity (89\%) and specificity (80\%).

\begin{tabular}{|l|c|c|l|c|c|}
\hline Variable & Sensitivity & Specificity & $\begin{array}{l}\text { +ve } \\
\text { predictive }\end{array}$ & $\begin{array}{l}\text {-ve } \\
\text { predictive }\end{array}$ & $\begin{array}{l}\text { Total } \\
\text { accuracy }\end{array}$ \\
\hline Isolated BPH & $89 \%$ & $80 \%$ & $59.8 \%$ & $87.5 \%$ & $88.9 \%$ \\
\hline Malignant & $100 \%$ & $81.1 \%$ & $61.6 \%$ & $100 \%$ & $90.3 \%$ \\
\hline BPH\& Prostatitis & $66.7 \%$ & $33.3 \%$ & $39.9 \%$ & $85 \%$ & $65.5 \%$ \\
\hline
\end{tabular}




\section{DISCUSSION}

Our study results match with those of Kwek et al, 2004 which showed that non invasive imaging techniques, such as T2- weighted MRI with an external pelvic array coil, have a good specificity (90\%). (4)

Also our results match with those of Bloch et al, 2007 that showed Overall staging of prostate cancer with T2-weighted magnetic resonance (MR) imaging at $1.5 \mathrm{~T}$ showing specificity values ranging from $67 \%$ to $87 \%$. (5)

Our study results also match with Turkbey et al, 2010 who included T2W, DW and DCE imaging, reported positive predictive values (PPVs) for multiparametric MRI's detection of prostate cancer in the overall prostate, $\mathrm{PZ}$, and $\mathrm{CG}$ to be $98 \%, 98 \%$, and $100 \%$, respectively. (6).

Also Delongchamps et al, 2011 reported that a combination of T2WI, DWI, and DCE imaging was significantly better at $\mathrm{PZ}$ tumor detection than eitherT2WI+DWI or T2WI alone. (7)

Haider et al, 2007 evaluating the addition of DWI to T2WI for the accuracy of prostate cancer detection, determined that DWI+T2WI was significantly more sensitive throughout the whole prostate compared to T2WI alone and was significantly more accurate in the PZ. (8)

\section{Another study done by Turkbey et al, 2010} using T2WI alone was compared to other modalities individually and to a combination of all three parameters revealed that Sensitivities were generally highest with T2WI while specificities were higher with DCE imaging. The study also demonstrated significant and additive improvement in the predictive values for prostate cancer detection in the $\mathrm{PZ}$ when different combinations of the modalities were combined, with the highest predictive value found using a combination of all three parameters. (6).
A study made by platzek et al, 2014 which showed that PI-RADS scores have limited prognostic value in regard to tumor grade, as the correlation of PI-RADS score and Gleason scores is low. The role of PI-RADS as an independent predictor of prognosis has yet to be evaluated. These results match with those of our study. (9).

Also Röthke1 et al 2013 reported that the PIRADS system gives the advantage of a standardized and easy communication of findings to other professional colleagues. For each lesion, a point score system between 1 and 5 is to be assigned per method. This is used to calculate the total score, which reflects the probability of the presence of clinically relevant cancer. The total score is then converted to the relevant PI-RADS score, providing the advantage that the final PIRADS score is independent of the number of techniques used and thus be easily communicated. (10)

\section{CONCULUSIONS:}

PIRADS as a scoring system can be used as a scoring system for every individual lesion and depending on its site. It can give a score for assessment of central zone lesions showing great advantage in assessment of cases where benign prostatic hyperplasia presents in individual with central zone carcinoma. PIRADS is an efficient way to help in the diagnosis that will result in better management and aids in guiding TRUS biopsy for better visualization of lesions prebiopsy leading to more accurate biopsy and accurate diagnosis. (PIRADS) is an efficient system for the diagnosis of different prostatic disease and depending on all sequences give better results than relying in the diagnosis on individual sequences.

\section{REFERENCES}

(1) Ferlay J, Shin HR, Bray F, et al. Globocan 2008 v1.2, Cancer incidence 
and mortality worldwide: IARC Cancer Base No. 10 [Internet]. Lyon, France: International Agency for Research on Cancer; 2010

(2) Kitzing YX, Prando A, Varol C, Karczmar GS, Maclean F, Oto A. Benign Conditions That Mimic Prostate Carcinoma: MR Imaging Features with Histopathologic Correlation. RadioGraphics. 2015 Nov 20;36(1):16275.

(3) Barentsz JO, Richenberg J, Clements R, Choyke P, Verma S, Villeirs , G, Rouviere O, Logager V, Fütterer JJ. ESUR prostate MR guidelines 2012. European Radiology. 2012 Apr 1;22(4):746-57.

(4) Kwek JW, Thng CH, Tan PH, Yuen JS, Khoo JB, Quek ST, Ho JT, Tan KP, Cheng CW. Phased-array magnetic resonance imaging of the prostate with correlation to radical prostatectomy specimens: local experience. Asian Journal of Surgery. 2004 Jul 31;27(3):219-24.

(5) Bloch BN, Furman-Haran E, Helbich TH, Lenkinski RE, Degani H, Kratzik C, Susani M, Haitel A, Jaromi S, Ngo L, Rofsky NM. Prostate cancer: accurate determination of extracapsular extension with high-spatial-resolution dynamic contrast-enhanced and T2weighted MR imaging — initial results 1 . Radiology. 2007 Oct;245(1):176-85.
(6) Turkbey B, Pinto PA, Mani H, Bernardo M, Pang Y, McKinney YL, Khurana K, Ravizzini GC, Albert PS, Merino MJ, Choyke PL. Prostate cancer: value of multiparametric $\mathrm{mr}$ imaging at $3 \mathrm{t}$ for detectionhistopathologifc correlation 1 . Radiology. 2010 Mar 10;255(1):89-99.

(7) Delongchamps NB, Beuvon F, Eiss D, Flam T, Muradyan N, Zerbib $M$, Peyromaure M, Cornud F. Multiparametric MRI is helpful to predict tumor focality, stage, and size in patients diagnosed with unilateral lowrisk prostate cancer. Prostate cancer and prostatic diseases. $2011 \quad$ Sep 1;14(3):232-7.

(8) Haider MA, van der Kwast TH, Tanguay J, Evans AJ, Hashmi AT, Lockwood G, Trachtenberg J. Combined T2-weighted and diffusionweighted MRI for localization of prostate cancer. American Journal of Roentgenology. 2007 Aug;189(2):323-8.

(9) Platzek I., Borkowetz A., Paulus T., Brauer T., Wirth M., Laniado M.; Dresden/DE PI-RADS classification: prognostic value for prostate cancer grading. European Congress of Radiology

2014.http://dx.doi.org/10.1594/ecr2014/ C-1622

(10) Roethke M, Blondin D, Schlemmer HP, Franiel T. PI-RADS classification: structured reporting for MRI of the prostate. Rofo. 2013 Mar 1;185(3):253-61. 